\title{
3 Researc Square

\section{Knowledge, Barriers, and Motivators to Cervical Cancer Screening in Rwanda: a Qualitative Study}

Jean Pierre Gafaranga ( $\nabla$ gafajepi@gmail.com )

University of Rwanda

Felix Manirakiza

Hamamatsu University School of Medicine

Emmanuel Ndagijimana

University of Rwanda

Jean Christian Ulimubabo

University Teaching Hospital of Kigali

David Irenée Karenzi

Ruhengeli Referral Hospital

Esperance Muhawenayo

University Teaching Hospital of Kigali

Phophina Gashugi

University Teaching Hospital of Kigali

Dancille Nyirasebura

University Teaching Hospital of Kigali

Belson Rugwizangoga

University Teaching Hospital of Kigali

\section{Research Article}

Keywords: cervical cancer, screening, fear for pain, qualitative, acceptance, Rwanda

Posted Date: September 27th, 2021

DOl: https://doi.org/10.21203/rs.3.rs-911914/v1

License: (c) (i) This work is licensed under a Creative Commons Attribution 4.0 International License.

Read Full License

Version of Record: A version of this preprint was published at International Journal of Women's Health on September 1st, 2022. See the published version at https://doi.org/10.2147/IJWH.S374487. 


\section{Abstract}

Background: Cervical cancer is a global public health problem with marked geographical disparity. There are high morbidity and mortality rates in low- and middle-income countries (LMICs) associated with low screening rates. In 2020, in Rwanda, 3.7 million women aged 15-59 years were at risk of developing cervical cancer. It is the most commonly diagnosed female cancer in Rwanda with an incidence rate of 42 cases per 100,000 women per year. Despite Rwanda being the first African country to vaccinate against human papillomavirus (HPV) with a three-dose regimen, vaccination coverage of nearly $93 \%$ in the target population of girls aged < 15 years, and having an established cervical cancer screening program, recent studies have found low screening rates. Our study sought to determine knowledge, motivators, and barriers to cervical cancer screening.

Methods: This was a qualitative phenomenological study conducted in an urban health facility (Muhima district hospital) and a rural health center (Nyagasambu health center) offering cervical screening services in Rwanda. Focus group discussion (FGD) participants were women seeking these services and other women attending the health facility for any reason, as well as female staff working in these health centers. We also interviewed a key informant (KI) from the national organization. FGD and KI interviews were recorded and transcribed, and the data were analyzed using thematic analysis.

Results: Thirty women were enrolled in the FGDs, with an average age of 39 years. Many FGD women showed knowledge about cervical cancer existence and prevention methods. However, fear for pain, lack of knowledge about screening, how and where the screening was done, and privacy concerns were recurring subthemes. Key-informant and some FGDs participants have also mentioned a lack of health insurance as a possible barrier for cervical cancer screening.

Conclusions: Barriers to the utilization of cervical cancer screening program in Rwanda are related to poor information about cervical cancer and the importance of screening among women, as well nonadherence to medical insurance schemes. Population sensitization through campaign and community outreach activities could have a positive impact on increasing the uptake of cervical cancer screening in Rwanda.

\section{Background}

Cancer incidence, morbidity, and mortality is still a major global public health challenge (1)(2)(3). Particularly, cervical cancer is a deadly disease, the fourth most common cancer in women worldwide with more than 604,127 new cases and 341,831 deaths in 2020 (1). Arbyn et al. reported that cervical cancer is among the top three cancers affecting women $<45$-year-old in $79 \%$ of 185 countries (4). Furthermore, according to GLOBOCAN 2020, cervical cancer is the most commonly diagnosed cancer in 23 countries and the leading cause of cancer-related deaths in 36 countries (1).

The disparity in cervical cancer incidence and mortality between developed and LMICs is highlighted by noting that 70 to $90 \%$ of cervical cancer cases and deaths occur in LMICs (4)(5)(6). Although considered 
a deadly disease, cervical cancer is one of the most easily managed forms of female cancers through screening, early detection, and treatment (1)(7)(8). However, screening rates are low in LMICs, especially in sub-Saharan Africa, compared to developed countries (9). East African countries, where Rwanda is located, have a high number of cervical cancer cases and deaths (4)(10)(11)(12). In addition, the majority

of women at risk for cervical cancer disease who reside in rural communities in LMICs may not have access to cervical cancer prevention programs (12); thus, only $0.4 \%-14 \%$ of rural women are screened, compared to $>20 \%$ of their urban counterparts(13).

In Rwanda, 3.7 million women aged 15-59 years, representing approximately $30 \%$ of the total population in 2020 (14), are at risk of developing cervical cancer (10). This is the most commonly diagnosed female cancer in Rwanda $(15,16)$ with a high incidence rate of 42 cases per 100,000 women per year $(16)$. A national program for cervical cancer prevention exists and the country has been the first African country to initiate a nationwide program to prevent cervical cancer through vaccination against human papillomavirus (HPV)(16). The program started in 2011 when girls in primary school grade six (approximately 12 years old) were vaccinated with quadrivalent (HPV16/18/6/11) HPV vaccine (16). The three-dose vaccination coverage was estimated at $93 \%$ of the target population (17).

Furthermore, Rwanda has introduced low-cost methods for cervical cancer screening including visual inspection of the cervix with acetic acid (VIA) and HPV-DNA test for women aged $\geq 35$ years with a repeat screening at 7 years for those with a negative result (15). Women with positive results are followed by colposcopy and biopsy (11).

Unfortunately, the cervical cancer screening rate had been low among Rwandan women. The reasons are not known (15). For example, in a study by Umulisa et al. (16) done between July 2013 and May 2014 only $2.6 \%$ of screened females reported previous participation in cervical cancer screening. This study aims to elucidate knowledge, barriers, and motivators that may affect Rwandan women in using cervical cancer screening services, to improve the overall utilization of this program and thus contribute to the prevention of cervical cancer.

\section{Methods}

We used a qualitative study approach to elucidate knowledge about cervical cancer, and identify barriers and motivators for Rwandan women to use cervical cancer screening services.

\section{Study population and settings}

The study was conducted in one urban (Muhima district hospital) and one rural health facility (Nyagasambu health center) which provided cervical cancer screening and involved women 30 to 50 years of age. These sites were selected based on the convenience method according to the locations (urban and rural areas), and the existence of screening services. 
We held FGD at two sites; the first site is Muhima district hospital in Kigali city. As a hospital serving women and children in an urban area, the hospital is committed to improving the health outcomes of women, children, and families from its catchment area, including providing cervical cancer screening services. The second site is Nyagasambu health center which is a primary health facility located in Gasabo district. The center is a community-based and patient-directed organization that delivers comprehensive, culturally competent, high-quality primary health care services. It integrates access to pharmacy, mental health, substance use disorder, and oral health services. Its services are used by ordinary citizens with a rural lifestyle. The FGD participants were recruited from the women who came to these sites for cervical cancer screening and for work-related activities, and women who accompanied patients to the sites.

We also conducted a key informant $(\mathrm{KI})$ survey at the national level to verify the implementation of the national cervical cancer program and how the system was expected to work in Rwanda. The interviewee was the national coordinator of the program.

\section{Data collection}

In FGD, we used a pre-tested semi-structured interview guide and conducted face-to-face FGD with the recording of the interview. The facilitators were trained on how to conduct an interview.

For the key informant survey, a pre-defined open-ended questionnaire was sent to the respondent who provided recorded answers to our questions. The lockdown period and COVID-19 preventive measures did not make possible an interview with the key informant.

\section{Data analysis}

The language used in FGD was Kinyarwanda which was the mother tongue of the participants. The recorded sessions were translated into the English language, transcribed, and verified for fidelity to the recording with two different investigators. In case of a difference in translation, these investigators made a consensus on the final translation with the assistance of the principal investigator. The observations were hand-coded and organized into themes using an inductive approach. We then detected regularities confirming the findings of predefined themes, which we then explored further and finally ended up with general conclusions.

During data analysis we followed the six steps described by Creswell (18):

Step 1: Data organization and preparation for analysis, during this step the audio recordings from interviews were transcribed into word document transcripts then translated into English.

Step 2: Reading through the data. Three investigators compiled the overall meaning to gain a general sense of the information and ideas that the participants provided.

Step 3: Start the coding process: To achieve this, sentences with similar meaning were colored with one marker and annotated using \# number, and rural or urban were added to each sentence to be able to 
know from which participant is coming from.

Step 4: Use the coding process to generate a description of the setting or people as well as categories or themes for analysis. At this step, we grouped similar subthemes under one theme regarding the predefined themes as this is a frequent practice in qualitative research related to the healthcare field.

Step 5: Advance how the description and themes will be represented in the qualitative narrative. We decided to use the narrative passage to be able to transmit the point of view/feelings from our respondents. Each narrative was annotated by the assigned \# number of the respondent, her living area (rural or urban), and her age.

Step 6: The final step consisted in interpreting our results by comparing our findings to the currently available data about cervical cancer screening and other healthcare-related issues.

The final report was reanalyzed, verified, and validated by all researchers.

\section{Results}

Five FGDs were conducted with 30 women (14 urban and 16 rural). In terms of the reasons for visiting the sites, 15 came specifically for cervical cancer screening, while the others came for other reasons ( 2 women working in the cleaning company, 2 women working as accountants, 6 women were accompanying their patients, 5 were there for other health reasons). The mean age was 39 years.

Qualitative data were classified into 3 different themes: knowledge about cervical cancer, barriers to the use or adoption of screening services, and motivation to use screening services. Each theme had subthemes as summarized in Table 1.

Table 1

Study themes

\begin{tabular}{|c|c|c|c|}
\hline Themes & Knowledge & Barriers & Motivators \\
\hline \multirow{4}{*}{$\begin{array}{l}\text { Sub- } \\
\text { themes }\end{array}$} & $\begin{array}{l}\text { - Existence of } \\
\text { cervical cancer }\end{array}$ & - Pain, fear to know the diagnosis & $\begin{array}{l}\text { - Government/healthcare } \\
\text { providers }\end{array}$ \\
\hline & $\begin{array}{l}\text { - Existence of } \\
\text { Prevention }\end{array}$ & - Feeling exposed and discomfort & $\begin{array}{l}\text { - Friends/family or history } \\
\text { of cancer }\end{array}$ \\
\hline & $\begin{array}{l}\text { - Existence of } \\
\text { Screening }\end{array}$ & - Financial/insurance issues & - Personal reason \\
\hline & & $\begin{array}{l}\text { - Other barriers (irregular services, } \\
\text { long-distance) }\end{array}$ & \\
\hline
\end{tabular}

\section{Knowledge about cervical cancer}




\section{Existence of cervical cancer}

The existence of cervical cancer was reported in all FGD. Women in the FDG showed awareness of risk factors, symptoms, and treatment options by the following expressions:

"Cervical cancer can mostly affect women in childbearing age...., or those who start having sex at an earlier age, among symptoms I know that someone may have persistent menses" (\#4, urban, age 45)

"Cervical cancer may be caused by becoming pregnant at an earlier age or by having too many babies," (\#1, urban, age 36)

"my friend had cervical cancer and told me that it is the most painful disease." (\#12, urban, age 33)

Examples of lack of knowledge about cervical cancer were expressed in statements such as:

"I did not know that cervical cancer is related to having sexual intercourse, ... I was thinking that it could be a disease like the most common disease" (\#2, urban, age 40).

Lack of knowledge can also be detected in such questions as:

Does cervical cancer have signs/symptoms that we can recognize and go for screening? (\#29, rural, age 37)

\section{Existence of cervical cancer prevention and screening}

Women understood how cervical cancer is screened. Some women also knew how cervical cancer could be prevented by using HPV vaccines, use of condoms, and limitation of the number of sex partners

"you can do prevention by not involving in prostitution, or by going for consultation as earlier as possible" (\#21, rural, age 46)

"you can use condoms when having sexual intercourse," (\#7, urban, age 34)

"I knew, through radio and television broadcast, that screening for cervical cancer is being done but I did not know where to go for screening." (\#9, urban, age 39)

\section{Barriers to cervical cancer screening:}

The major barriers identified include the following:

1. Fear of pain, especially during speculum insertion and removal:

"I thought about going to screening many times, but I always had fear of pain, ... even in the past time I went to a screening area where the campaign for cervical cancer screening was being done but because of fear of the pain I did not yet participate in screening" (\#13, urban, age 40) 
"I always had fear of pain even though today I came for screening, I come with reluctance because of fear for pain" (\#6, urban, age 38)

"I thought about going for screening but once I heard that it is painful, I decided not to go (\#19, rural, age 36)

"I know nothing about cervical cancer screening but those who went for it told me that the procedure is painful". (\#2, urban, age 40)

Concerning fear for the pain two women suggested that:

"I can suggest the use of anaesthesia during screening because the procedure is painful, even though I do not know if it is possible to use anaesthesia" (\#10, urban, age 39)

"I am afraid that the procedure is painful and I am asking myself if there is no other way of screening for cervical cancer, for example by using imaging tool or ultrasound" (\#17 rural, age 46)

2. Privacy concerns especially discomfort with exposure of private parts during the procedure:

"Let's consider a not yet married female (a girl) with no prior experience of giving childbirth, she is very concerned with her privacy, she may have shyness, ... There is no other way of doing screening without exposing private part of the body?" (\#26, rural, age 58)

Another woman asked about alternative procedures that would not require exposure.

"Can you use other technics for screening like ultrasound or by testing through blood?" (\#6, urban, age 38)

3. Financial issues which prevented them from screening, such as the lack of health insurance, unaffordable consultation fees, and other medical expenses.

"I had a desire for screening but because of lack of health insurance, I waited until I got it" (\#11, urban, age 41)

4. Other barriers include the long-distance from the testing site, lack of services nearby, inadequate information about the location of screening services, and other administrative problems such as not having enough testing materials and confusion about screening eligibility

"When I reached the screening service, they told me that the screening tools were not available and they advised me to come back at another time" (\#23, rural, age 35)

"I had a desire for cervical cancer screening for a long time but I could not know where I can get screening services" (\#9, urban, age 39) 
"I came for cervical cancer screening in past time but nurses told me that they can not do screening for me because I was less than 30 years old. Now as I have reached 30 years, I am back at the health center and am happy that now they will do a screening for me!"(\#22, rural, age 30)

Another woman was afraid that she might be too old because she was 59 years old, but was reassured by the nurse that she was eligible to be screened.

None of the women expressed religious beliefs or familial reasons as barriers to attending cancer screening services. However, one female insisted that she would not attend cervical cancer screening because she was a widow:

"Because I do not have a husband ... and as are the males who bring cancer to the females... and I had my way, I do abstinence, therefore, I think that there is no reason that I have to go for screening, but in case I have signs/symptoms I will go for consultation" (\#30, rural, age 46.)

\section{Motivators for use of cervical cancer screening services Government and healthcare providers' role}

The current study found that the main motivator cited for using screening services was from government promotion through different channels including mobile phones, radios and television broadcasts, and local leaders. Individual health care providers also helped.

"Even today I received an SMS (short message service) advertising for cervical cancer screening" (24, rural, age 42)

"When we come for antenatal care or the health of our children, nurses sensitize us about cervical cancer screening," (\#7, urban, age 34)

\section{Friends and family members or familial history of cancer}

Women whose family members or relatives or friends had previously been screened for cervical cancer or had a history of cancer were motivated to seek screening services.

"I come because I had my grand sister who had cervical cancer" (\# 25, rural, age 42)

"My friend had many times advised me to go for cervical cancer screening."(\#10, urban, age 39)

"My daughter has cervical cancer before she did not know that it was cancer and she was seriously ill weighing like $20 \mathrm{~kg}$ but after we consult different hospitals, she received treatment and she is doing well with about more than $50 \mathrm{~kg}$, she is now healthy". (\# 26., rural, age 58)

"After my mother died of cervical cancer, I thought about cervical cancer screening but I did not yet come because I did not have insurance."(\#18, rural, age 36)

Personal reason: early treatment and quick recovery 
A few women reported that they decided to go for cervical cancer screening on their own without any external motivators. They thought that they were increasing the recovery chances if the results were positive.

"When you go for screening, if you have illness they will treat you as earlier as possible and you will be cured. And if they find that you do not have cancer, they will teach you more about how to do prevention" (\# 5, urban, age 46)

\section{Cervical cancer screening in Rwanda: insight from the key informant.}

We identified one senior officer from the non-communicable diseases division of Rwanda biomedical center of the Ministry of health who served as a key informant. His views are summarized below

\section{The current situation on cervical cancer screening}

"The statistics show that we still have a low number of people who come for cervical cancer screening. But the screening service is also at its earliest stage. We have now done training of health care providers in half of the health centers countrywide. In 2013 we did a screening campaign in 5 out of 30 districts and we did a screening of 10,000 people using HPV testing kits. In 2014 we changed our approach to using visual inspection of the cervix with acetic acid (VIA) because of a financial barrier. As VIA needs further training, we did a training until 2018.

Since 2020, besides VIA we are also using HPV DNA test for which we started with 3 districts. If a test is positive, we do VIA and if positive we do treatment with thermo-ablation. The most challenge is the low rate of training of health personnel because you can not avail devices without trained people to use them. And we need to train people to be able to do the basic treatment (thermo-ablation) of VIA positive cases at health center level".

\section{Barriers to reaching the goal}

Among the major barriers mentioned by the key informant were the issues of lab consumables and reagents as screening services are not covered by health insurance companies.

"We are also facing the issue of consumables/reagents because screening services are not reimbursed by health insurance companies in our country. But we are doing our best to work together with insurance companies and explain to them why it is better to invest in screening services: they have to think about the price of screening which is around 1000-1500RWF (1 to 1.5 USD) and the price that they will pay in future if someone is having cervical cancer because she has not been screened. The major message is that insurance companies must not think about the premium of the client within one year of insurance but within 30 years later on".

\section{Key factors for more success}


When queried about key factors to make screening program successful, two main important ideas were raised:

'We need collaboration from lower level to high level..., Cervical cancer screening campaign approach looks more productive/ effective than routine screening because for routine screening women are procrastinating due to their daily work. Campaign periods target a lot of people but we use HPV DNA test which cost 8-15 USD for one female and this cost is high as we target about 1.5 million for the entire country to reach the World health organization (WHO)'s target of cervical cancer elimination by 2030".

We investigated how the number of women attending /seeking cervical cancer screening services can be increased and if there is no problem with the referral system and we found that women have the will to attend these services but the problem could be found elsewhere as it is stated below:

"The problem is not limited to females only because they are eager to come for screening especially during community outreach strategies and females transmit the message to their neighbours/ friends. Therefore, there is a need to avail services at their nearest health facilities and involve community health workers (CHW) and media to increase the awareness of cancer existence, prevention, and screening program. We must also increase the number of trained health care providers, number of community outreach activities because, during this period, females attend the screening services with a reduction of cost and do all possible to attend the screening.

\section{Discussion}

In this qualitative study, most women in the focus groups had some knowledge about cervical cancer and the screening methods used. We can speculate that the high rate of awareness about cervical cancer may be associated with health promotion efforts using SMS, telecommunication, and radio station broadcast, which were mentioned many times during the focus group discussions.

Fear of pain, concern about lack of privacy, and financial costs were 3 major barriers cited for not coming forward for cervical cancer screening. Fear of pain, which can also be coupled with shyness or uncertainty for privacy has been previously reported in Africa, especially Tanzania which is a neighbouring country of Rwanda (19)(20). As there is increasing evidence of the effectiveness and acceptability of self-sampling in cervical cancer screening and diagnostic testing of diseases of the female genital tract (21)(19) this might be a remedy for both the concern of lack of privacy and pain as barriers for attending cervical cancer screening. The inability to cover the expenses of cervical cancer screening in rural African settings was reported as a major finding in the systematic literature review by Rahman et al. (20). In our study, participants reported that health insurance did not cover some of the costs related to screening services. Other women noted that they waited until they had health insurance before attendance at screening services.

While someone may expect familial and religious reasons to be among barriers (20), in our study none of the women raised them. Long-distance as a barrier has been reported by other authors; hence community 
outreach and or use of community health workers may be needed to address this problem. We suggest involving community health workers because they are playing a considerable role in the healthcare system in Rwanda (22) and have been used in different roles for cervical cancer screening elsewhere (23).

Community outreach activities were mentioned by some women as the source of information about cervical cancer and by the key informant as one way of increasing coverage for cancer screening. We, therefore, think that community outreach aiming at sensitization, teaching, and screening for cervical cancer should be considered as a remedy for both long-distance, lack of information, and procrastination. Additionally, community outreach has been found to increase the cervical cancer screening rate in the United States of America (24)(25).

\section{Limitation}

Our study has some limitations, which further research may be able to address. First of all, the majority of our respondents came from the ones who had access to health services. We did not go out to women in the community in either urban or rural settings. Future research might involve the women who are using herbal medicine or religious approach to keep themselves safe. In addition, poverty is well known to interfere with healthcare-seeking behaviour (26-28) and we only interviewed women who had come to a health facility.

\section{Conclusion}

This study has found fear for pain, privacy concerns, and cost as major barriers for not coming for cervical cancer screening. Additionally, lack of information and misinformation may be contributing to the low rate of participation in this program. On the other hand, the use of government sensitization campaigns, health workers' recommendations, and community outreach activities could have a positive impact on increasing the uptake of cervical cancer screening.

\section{Abbreviations}

CHW: Community health workers

CMHS: College of medicine and health sciences

COVID-19: Coronavirus disease -2019

DNA: Deoxyribonucleic acid

FGD: Focus group discussion

HPV: Human papilloma virus 
IRB: Institution review board

Kl: key informant

LMICs: Low -and -middle-income countries

RWF: Rwandan franc

SMS: short message service

USD: United States dollar

VIA: Visual inspection of the cervix with acetic acid

WHO: World health organization

\section{Declarations}

\section{Ethics approval and consent to participate}

The study was approved by the institutional review board (IRB) of the University of Rwanda College of Medicine and Health Sciences (IRB Notice No 524/CMHS IRB/2019). All focus group discussions participants as well as the key informant gave written informed consent forms before participating in the discussion/survey. Focus group interviews and key informant survey were carried out in accordance with Rwandan national guidelines and regulations and in accordance with the Helsinki declaration.

\section{Consent for publication}

Not applicable

\section{Availability of data and materials}

The audio data that support the findings of this study are not publicly available because they contain information that could compromise research participants' privacy/consent. The transcribed and translated data are available from the corresponding author upon reasonable request.

\section{Competing interests}

The authors declare that they have no competing interests

\section{Funding}

This study has received a grant from Micro Research Project/Dalhousie University, IWK Health Centre, 5850 /5980 University Ave, Halifax, Nova Scotia, B3K 6R8, Canada.

\section{Authors' contributions}


JPG, FM: conceptualization, methodology, formal analysis, project administration, writing - original draft. EN: methodology, formal analysis, writing - original draft.

JCU, BR: conceptualization, supervision, methodology, data acquisition, reviewing - original draft IDK, EM, PG, DN: conceptualization, methodology, data acquisition, reviewing - original draft

\section{Acknowledgments}

We acknowledge Micro Research Project/Dalhousie University, IWK Health Centre, 5850/5980 University Ave, Halifax, Nova Scotia, B3K 6R8, Canada, MicroResearch International (www.microresearch.ca) for providing mentorship in the development and writing up of the findings.

\section{References}

1. Sung H, Ferlay J, Siegel RL, Laversanne M, Soerjomataram I, Jemal A, et al. Global cancer statistics 2020: GLOBOCAN estimates of incidence and mortality worldwide for 36 cancers in 185 countries. CA Cancer J Clin. 2021;0(0):1-41.

2. World Health Organization. Global Status Report On Noncommunicable Diseases 2014. 2014;

3. World Health Organisation (WHO). Non-communicable diseases country profiles 2018. Geneva; 2018.

4. Arbyn M, Weiderpass E, Bruni L, de Sanjosé S, Saraiya M, Ferlay J, et al. Estimates of incidence and mortality of cervical cancer in 2018: a worldwide analysis. Lancet Glob Heal. 2020;8(2):e191-203.

5. Chuang LT, Temin S, Camacho R, Dueñas-Gonzalez A, Feldman S, Gultekin M, et al. Management and Care of Women With Invasive Cervical Cancer: American Society of Clinical Oncology ResourceStratified Clinical Practice Guideline. J Glob Oncol. 2016;2(5):311-40.

6. WHO. Human papillomavirus ( HPV) and cervical cancer Key facts How HPV infection leads to cervical cancer [Internet]. World Health Organization. 2020 [cited 2021 Mar 10]. Available from: https://www.who.int/news-room/fact-sheets/detail/human-papillomavirus-(hpv)-and-cervical-cancer

7. World Health Organisation (WHO). Draft: Global Strategy Towards the Elimination of Cervical Cancer As A Public Health Problem. 2019;(December). Available from:

https://www.who.int/publications/i/item/9789240014107\%0Ahttps://www.who.int/docs/defaultsource/documents/cervical-cancer-elimination-draft-strategy.pdf

8. WHO technical guidance and specifications of medical devices for screening and treatment of precancerous lesions in the prevention of cervical cancer. Geneva; 2020.

9. Lemp JM, De Neve JW, Bussmann H, Chen S, Manne-Goehler J, Theilmann M, et al. Lifetime Prevalence of Cervical Cancer Screening in 55 Low-and Middle-Income Countries. JAMA - J Am Med Assoc. 2020;324(15):1532-42.

10. Aweke YH, Ayanto SY, Ersado TL. Knowledge, attitude and practice for cervical cancer prevention and control among women of childbearing age in Hossana Town, Hadiya zone, Southern Ethiopia: 
Community-based cross-sectional study. PLoS One [Internet]. 2017;12(7). Available from: http://dx.doi.org/10.1371/journal.pone.0181415

11. Binagwaho A, Ngabo F, Wagner CM, Mugeni C, Gatera M, Nutt CT, et al. Integration of comprehensive women's health programmes into health systems: cervical cancer prevention, care and control in Rwanda. Bull World Health Organ. 2013;91(9):697-703.

12. LaVigne AW, Triedman SA, Randall TC, Trimble EL, Viswanathan AN. Cervical cancer in low and middle income countries: Addressing barriers to radiotherapy delivery. Gynecol Oncol Reports [Internet]. 2017;22(May):16-20. Available from: https://doi.org/10.1016/j.gore.2017.08.004

13. Onyenwenyi AOC, Gugu GM. Strategies for the prevention and control of cervical cancer in rural communities: a Nigerian perspective. J Community Med Prim Heal Care. 2016;28(2):77-93.

14. Report T. National Institute of Statistics of Rwanda. Fourth Population and Housing Census, Rwanda, 2012. Thematic Report. Population Projections. [Internet] [Internet]. 2014;11. Available from: https://www.statistics.gov.rw/publication/rphc4-population-projections

15. Ruzigana G, Bazzet-Matabele L, Rulisa S, Martin AN, Ghebre RG. Cervical cancer screening at a tertiary care center in Rwanda. Gynecol Oncol Reports. 2017;21(May):13-6.

16. Umulisa MC, Franceschi S, Baussano I, Tenet V, Uwimbabazi M, Rugwizangoga B, et al. Evaluation of human-papillomavirus testing and visual inspection for cervical cancer screening in Rwanda. BMC Womens Health. 2018;18(1):1-8.

17. Ngabo F, Franceschi S, Baussano I, Umulisa MC, Snijders PJF, Uyterlinde AM, et al. Human papillomavirus infection in Rwanda at the moment of implementation of a national HPV vaccination programme. BMC Infect Dis [Internet]. 2016;16(1):1-10. Available from:

http://dx.doi.org/10.1186/s12879-016-1539-6

18. Creswell JW. Research design. Qualitative, quantitative and mixed methods approaches. 4th ed. SAGE; 2014. 245-250 p.

19. Bakiewicz A, Rasch V, Mwaiselage J, Linde DS. 'The best thing is that you are doing it for yourself' Perspectives on acceptability and feasibility of HPV self-sampling among cervical cancer screening clients in Tanzania: A qualitative pilot study. BMC Womens Health. 2020;20(1):1-9.

20. Rahman R, Clark MD, Collins Z, Traore F, Dioukhane EM, Thiam H, et al. Cervical cancer screening decentralized policy adaptation: an African rural-context-specific systematic literature review. Glob Health Action [Internet]. 2019;12(1):1-14. Available from:

https://doi.org/10.1080/16549716.2019.1587894

21. Ndayisaba G, Verwijs MC, Van Eeckhoudt S, Gasarabwe A, Hardy L, Borgdorff H, et al. Feasibility and acceptability of a novel cervicovaginal lavage self-sampling device among women in kigali, Rwanda. Sex Transm Dis. 2013;40(7):552-5.

22. Lauren C. Rwanda 's Community Health Worker Program [Internet]. [cited 2021 Jan 7]. Available from: https://chwcentral.org/rwandas-community-health-worker-progam/

23. O'Donovan J, O'Donovan C, Nagraj S. The role of community health workers in cervical cancer screening in low-income and middle-income countries: A systematic scoping review of the literature. 
BMJ Glob Heal. 2019;4(3):1-8.

24. Castle PE, Rausa A, Walls T, Gravitt PE, Partridge EE, Olivo V, et al. Comparative community outreach to increase cervical cancer screening in the Mississippi Delta. Prev Med (Baltim) [Internet]. 2011 Jun;52(6):452-5. Available from: https://linkinghub.elsevier.com/retrieve/pii/S0091743511001290

25. Levano W, Miller JW, Leonard B, Bellick L, Crane BE, Kennedy SK, et al. Public education and targeted outreach to underserved women through the National Breast and Cervical Cancer Early Detection Program. Cancer. 2014;120(SUPPL. 16):2591-6.

26. Oduro Appiah J, Agyemang-Duah W, Fordjour AA, Adei D. Barriers to formal healthcare utilisation among poor older people under the Livelihood Empowerment Against Poverty Programme in Ghana. BMC Public Health. 2019;19(1):1-12.

27. Masiye $F$, Kaonga $O$. Determinants of healthcare utilisation and out-of-pocket payments in the context of free public primary healthcare in Zambia. Int J Heal Policy Manag [Internet]. 2016;5(12):693-703. Available from: http://dx.doi.org/10.15171/ijhpm.2016.65

28. Ponsar F, Tayler-Smith K, Philips M, Gerard S, Van Herp M, Reid T, et al. No cash, no care: How user fees endanger health-lessons learnt regarding financial barriers to healthcare services in Burundi, Sierra Leone, Democratic Republic of Congo, Chad, Haiti and Mali. Int Health [Internet]. 2011;3(2):91100. Available from: http://dx.doi.org/10.1016/j.inhe.2011.01.002 